\title{
Songbird Responses to Land Preservation Within Southern New England Cluster Subdivisions
}

\author{
Kenneth Bryan Raposa ${ }^{1}$, Richard A. McKinney ${ }^{2} \&$ Scott Millar $^{3}$ \\ ${ }^{1}$ Narragansett Bay National Estuarine Research Reserve, Rhode Island, USA \\ ${ }^{2}$ Atlantic Ecology Division, US Environmental Protection Agency, Rhode Island, USA \\ 3 Division of Planning and Development, Rhode Island Department of Environmental Management, Rhode \\ Island, USA \\ Correspondence: Kenneth B. Raposa, Narragansett Bay National Estuarine Research Reserve, Prudence Island, \\ RI 02872, USA. Tel: 1-401-683-7849. E-mail: Kenny@nbnerr.org
}

Received: July 10, 2013 Accepted: September 23, 2013 Online Published: October 10, 2013

doi:10.5539/jsd.v6n11p26 URL: http://dx.doi.org/10.5539/jsd.v6n11p26

\begin{abstract}
Cluster subdivisions were designed to protect open space in response to rapid rates of conventional development. One of the proclaimed benefits of preserving open space within cluster subdivisions is the provision of habitat for native wildlife, but this has rarely been evaluated. This study examined songbird response to the amount of land protected within cluster subdivisions in Rhode Island (USA). We selected 11 sites along a gradient based on the relative amount of land protected within a site (\% land under a conservation easement; \%CE). We used nonparametric multivariate statistics to compare songbird communities between protected and developed areas within subdivisions and regression analyses to relate bird abundance and community metrics to \%CE. Songbird communities differed significantly between protected and developed areas within cluster subdivisions. Songbird richness and diversity both peaked between 73-74\% $\%$, while densities of forest interior and human intolerant species increased with increasing \%CE. Ovenbird, Veery, and Pine Warbler most typified high \%CE sites and were found most often in protected areas far from development edges. This study demonstrates that cluster subdivisions need to preserve approximately $70-75 \%$ of the original undeveloped parcel of land in order to maximize songbird diversity. A higher percentage should be preserved in large contiguous blocks to further benefit forest interior species. This suggests that proposed regulations that require Rhode Island subdivisions to protect at least $50 \%$ of a parcel's buildable land may not be adequate to enhance bird diversity or preserve species that depend on large contiguous blocks of forest interior habitat.
\end{abstract}

Keywords: songbirds, cluster subdivisions, New England, habitat value, diversity, conservation easements

\section{Introduction}

A defining characteristic of the changing United States landscape is the rapid and ongoing loss of natural and agricultural habitats to residential and urban development. The area of developed lands increased by approximately 14.2 million ha (48\%) between 1982 and 2003 (White, Morzillo, \& Alig, 2009) and as of 2007 comprised $6 \%$ of the landmass of the conterminous United States (United States Department of Agriculture [USDA], 2009). Although estimates vary, similar rates of development are expected to continue. For example, Stein et al. (2005) estimated that approximately 18 million ha of additional land will be developed by 2030 , while White et al. (2009) projected that 22 million ha of land will be developed between 2003 and 2030. Much of this development has occurred (and will occur) in exurban areas, or areas beyond urban centers and their suburbs, through the conversion of natural and agricultural habitats into residential housing (Brown, Johnson, Loveland, \& Theobald, 2005; Radeloff, Hammer, \& Stewart, 2005; Theobald, 2005). This exorbitant growth has many negative ecological implications, including the loss and fragmentation of natural habitats (Ritters et al., 2002; Radeloff et al., 2005; Drummond \& Loveland, 2010), reduced air and water quality (Tu, Xia, Clark, \& Grei, 2007; Duh, Shandas, Chang, \& George, 2008; Stone, 2008), declines and extinctions of native and rare species (Czech, Krausman, \& Devers, 2000; Marzluff, 2001; McKinney, 2006), introduction and expansion of non-native and invasive species (Riley et al., 2005; McKinney, 2006), and disruption of natural ecological processes (e.g., fire regimes) (Syphard et al., 2007). 
Much of the loss of exurban habitats is due to the proliferation and sprawl of conventional or tract subdivisions resulting from suburban zoning and subdivision ordinances. These kinds of residential developments were typically comprised of large housing lots with little or no preservation of natural habitats as open space (Arendt, 1994; Flinker, 2003). The concept of cluster subdivisions was developed in response to growing concern over the rapid loss of natural habitats from conventional development practices. Cluster subdivisions are designed specifically to allow a similar number of housing units as conventional developments in a given area, but by reducing the individual lot size (typically less than 1 acre) and grouping houses together, a portion of the original buildable parcel is protected as open space. The potential benefits of cluster subdivisions include the retention of some of the original character of the landscape, a more aesthetically pleasing landscape, increased property tax revenues, and the provision of open space and habitat for both human residents and native plants and wildlife (Brabec, 1994; Flinker, 2003; Odell, Theobald, \& Knight, 2003). In practice, however, not all of these benefits are realized and the value of cluster subdivisions remains debatable (Arendt, 1996; Brabec, 2001; Lenth, Knight, \& Gilbert, 2006; Freeman \& Bell, 2011).

The idea that native wildlife will benefit when natural habitats are protected within cluster subdivisions is often cited (Arendt, 1996; Theobald, Miller, \& Hobbs, 1997; Odell et al., 2003). On a larger scale, it is clear that remnant or protected fragments of natural habitats within urbanizing landscapes can provide important habitat for native and other human-intolerant species (Donnelly \& Marzluff, 2004; Fernández-Juricic, 2004; Chace \& Walsh, 2006; Mason, Moorman, Hess, \& Sinclair, 2007; Oliver et al., 2011). However, the same may not be true at the scale of individual cluster subdivisions, although published case studies are severely lacking. In the sole study of which we are aware that directly quantified the value of cluster subdivisions for wildlife, Lenth et al. (2006) found that in developments in mixed-grass prairie ecosystems in Colorado, plants, birds, and mammals were generally similar between cluster and conventional subdivisions. While their findings call into question the general wildlife-benefit assumption associated with cluster subdivisions, it is clear that further studies are needed to provide reliable information about the ecological effects of development alternatives.

Rhode Island is a microcosm of the patterns that are occurring nationally in terms of urbanization and the construction of cluster subdivisions without fully understanding their ecological effects. As of 1997, 33\% of non-federal rural land in Rhode Island was developed, placing it second only to New Jersey (White et al., 2009). Developed lands in Rhode Island increased by 43\% between 1970 and 1995 with most of this development occurring in rural and exurban areas (Rhode Island Statewide Planning Program [RISPP], 2006). Suburban sprawl and residential housing development in Rhode Island have increased the amount of impervious surfaces and led to forest fragmentation and loss (Novak \& Wang, 2004; Zhou \& Wang, 2007). In an attempt to help slow urban and suburban sprawl and the loss of natural and agricultural areas, 19 of Rhode Island's 39 towns had passed ordinances to allow cluster subdivisions (or similar alternative development types) by 1990 (RISPP, 2001). This increased to 28 towns by 2000 , five of which mandated that new subdivisions must follow cluster subdivision guidelines (RISPP, 2001). In suburban North Kingstown RI, for example, 37 cluster subdivisions, comprising 976 hectares (approximately 6.5\% of the entire town), had been built as of 2008 (Town of North Kingstown, unpublished data). However, the economic, ecological and aesthetic effects of cluster subdivisions in Rhode Island have not been quantified and, specifically, the value of open space that is protected within cluster subdivisions for wildlife remains unknown.

The overall goal of this study was to quantify the value of protected open space within Rhode Island cluster subdivisions for songbird populations and communities. More specifically, this study 1) directly compared songbirds between developed and protected areas within cluster subdivisions, and 2) examined songbird species, guild, and community metrics along a gradient based on the relative amount of land that was conserved within cluster subdivisions. The former goal will help quantify the value of natural habitats that are protected within cluster subdivisions for songbirds. The latter goal will help determine the proportion of the original undeveloped parcel of land that should be protected to provide the most benefit for songbirds.

\section{Methods}

\subsection{Study Sites}

This study was conducted at eleven sites that represent a gradient based on the relative amount of land that is protected with conservation easements (\%CE; percent land under a conservation easement). Nine of the sites were cluster subdivisions with varying amounts of \%CE. These were augmented with one conventional development site $(0 \% \mathrm{CE})$ and one undeveloped state forest $(100 \% \mathrm{CE})$, which represent the two endpoints along the \%CE gradient. Cluster sites were selected from a pool of 37 cluster subdivisions comprising 976 hectares based on comparable size, accessibility of conservation easement areas, and proximity to one another 
(to minimize variability in regional bird species assemblages), and then targeted to ensure spread along the $\% \mathrm{CE}$ gradient (Table 1). All of the subdivisions and the state forest were located within the town of North Kingstown, RI, but due to a lack of appropriate sites, the conventional development site was located within the neighboring town of Narragansett, RI. However, both towns are classified as suburban (RISPP, 1999) and all sites were located within $17 \mathrm{~km}$ of each other (Figure 1). All sites were selected from suburban areas in order to control for any potentially confounding effects from different surrounding matrix types. Percent CE was calculated by dividing the area of conserved land within the original undeveloped parcel by total parcel size (Table 1; Figure 2 ). The density of edges between conservation easement and developed areas at each site was calculated by dividing the total length of these types of edges by the total area of the site (Table 1). Based on 2003/2004 land use/land cover data from the Rhode Island Geographic Information System (RIGIS, 2007), conservation easement lands within the nine subdivisions were dominated by deciduous and mixed forests $(90.0 \%)$, followed by residential development $(3.5 \%)$, wetlands and water $(3.3 \%)$, power line easements $(1.6 \%)$, pasture $(1.1 \%)$, and other minor habitat types $(0.4 \%)$.

Table 1. Characteristics of the eleven sites included in this study. \%CE for each site is the area protected within conservation easements divided by the area of the entire site multiplied by 100 . Edge density for each site is the length of edge between easement and non-easement areas divided by total site area

\begin{tabular}{|c|c|c|c|c|c|c|c|}
\hline Site Name & $\begin{array}{l}\text { Site } \\
\text { code }\end{array}$ & Type & Age & $\begin{array}{c}\text { Total } \\
\text { area (ha) }\end{array}$ & $\begin{array}{l}\text { Conservation } \\
\text { Easement (ha) }\end{array}$ & $\% \mathrm{CE}$ & $\begin{array}{l}\text { Edge density } \\
\qquad\left(\mathrm{m} \mathrm{ha}^{-1}\right)\end{array}$ \\
\hline Candy Apple & CAA & Cluster & 1996 & 41.95 & 31.89 & 76.0 & 69.05 \\
\hline Carriage Hill & $\mathrm{CAH}$ & Cluster & 1981 & 33.55 & 21.39 & 63.8 & 82.79 \\
\hline Cocumcussoc & $\mathrm{COC}$ & State Forest & $\mathrm{n} / \mathrm{a}$ & $\mathrm{n} / \mathrm{a}^{\mathrm{a}}$ & $\mathrm{n} / \mathrm{a}^{\mathrm{a}}$ & 100.0 & $\mathrm{n} / \mathrm{a}$ \\
\hline Cole Drive & COD & Cluster & 1985 & 26.08 & 17.16 & 65.8 & 148.70 \\
\hline Laurel Ridge & LAR & Cluster & 1988 & 33.68 & 13.62 & 40.4 & 157.67 \\
\hline Mettatuxet & MET & Conventional & variable & $\mathrm{n} / \mathrm{a}^{\mathrm{b}}$ & 0.00 & 0.0 & $\mathrm{n} / \mathrm{a}$ \\
\hline $\begin{array}{c}\text { Misty } \\
\text { Meadows }\end{array}$ & MIM & Cluster & 1999 & 42.23 & 35.43 & 83.9 & 53.75 \\
\hline $\begin{array}{l}\text { Pride's } \\
\text { Crossing }\end{array}$ & PRC & Cluster & 2000 & 22.93 & 16.65 & 72.6 & 51.19 \\
\hline Shady Lea & SHL & Cluster & 1994-96 & 40.84 & 30.58 & 74.9 & 57.82 \\
\hline Signal Rock & SIR & Cluster & 1993 & 42.97 & 29.42 & 68.5 & 132.87 \\
\hline $\begin{array}{l}\text { Orchard } \\
\text { Woods }\end{array}$ & ORE & Cluster & 2001 & 25.04 & 14.93 & 59.6 & 79.92 \\
\hline
\end{tabular}

${ }^{\mathrm{a}}$ The total size of COC is 158 ha; we selected stations within a central area of 34 ha, which represents the mean size of the nine cluster subdivisions.

${ }^{\mathrm{b}}$ MET is a large conventional development that does not have distinct boundaries; we selected stations within a central area of 34 acres, which represents the mean size of the nine cluster subdivisions. 


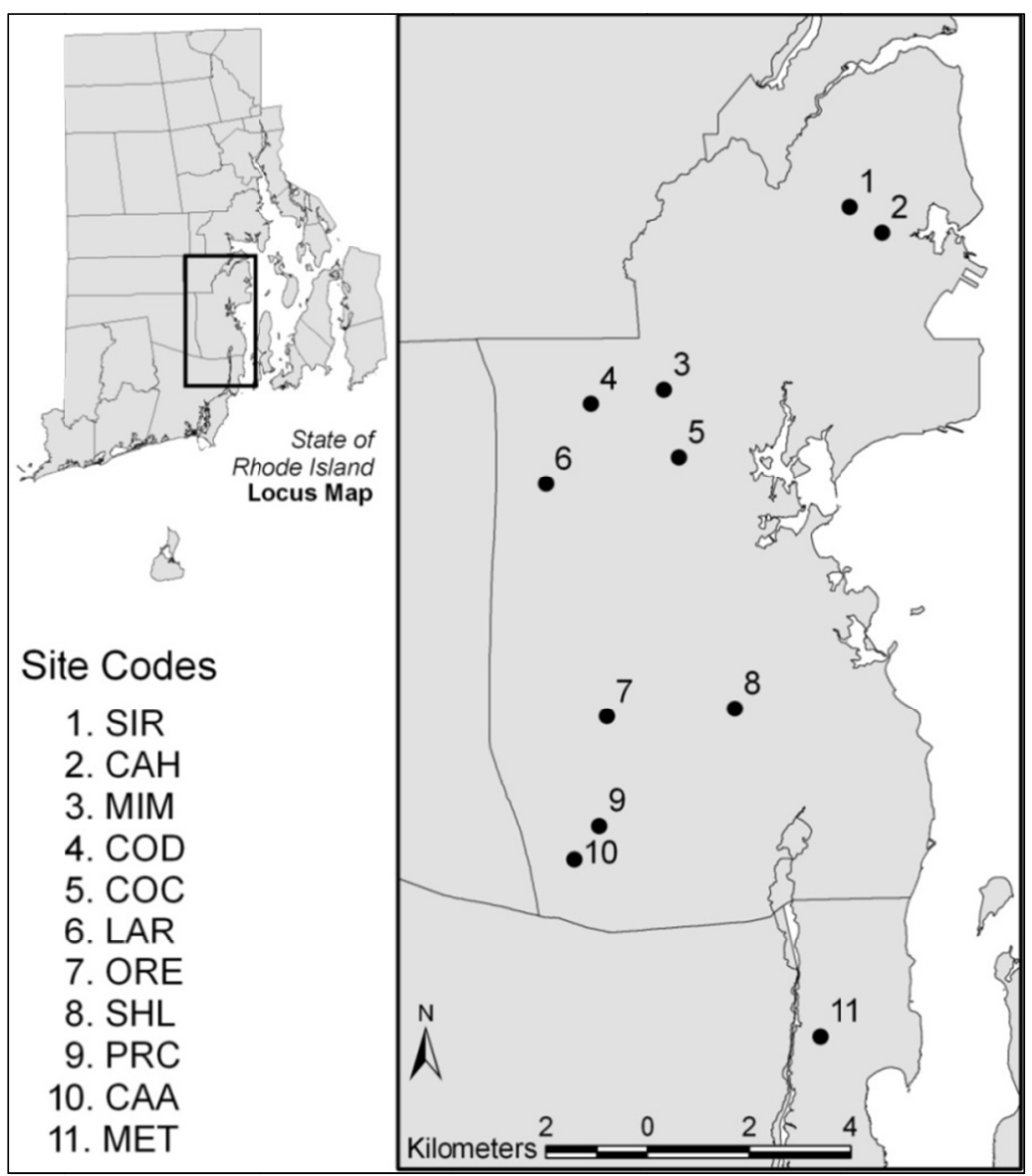

Figure 1. Locations and three-letter codes of the 11 sites included in this study (refer to Table 1 for all site codes and names)

Note: All sites are located in North Kingstown, RI except Metatuxet (MET; Site 11), which is in Narragansett, RI. Lines represent boundaries between towns.

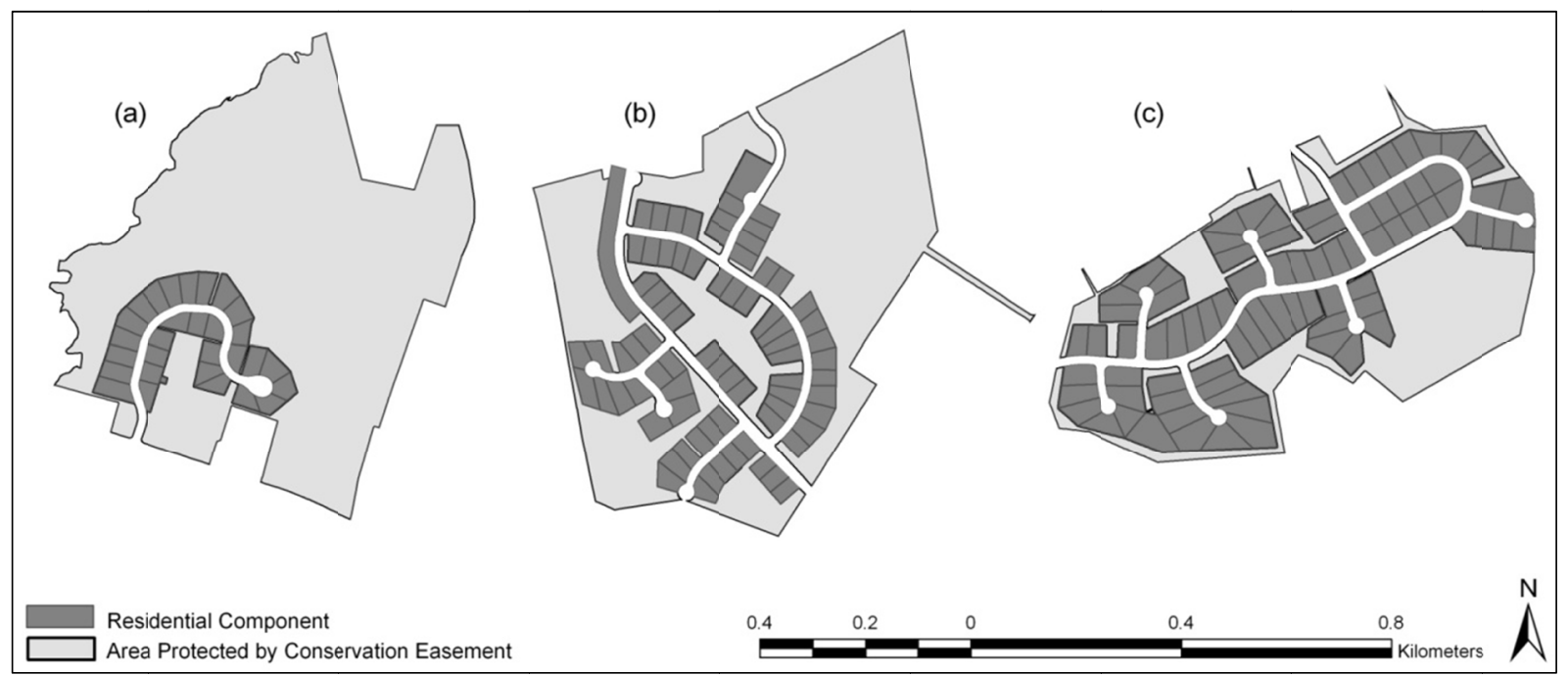

Figure 2. Three examples of cluster subdivisions with varying amounts of $\% \mathrm{CE}$

Note: A=Misty Meadows (MIM; 84 \%CE); B=Signal Rock (SIR; 69 \%CE); C=Laurel Ridge (LAR; $40 \% \mathrm{CE}$ ). These figures were created with the parcels dataset provided by the Town of North Kingstown, RI and with the municipal and NGO conservation lands coverage available from the Rhode Island Geographic Information System (RIGIS, 2011). 


\subsection{Data Collection}

We randomly selected 7-8 sampling stations within each of the 11 sites. All stations were selected using ARCGIS (v. 10) prior to initiating field sampling. Stations were spaced a minimum of $200 \mathrm{~m}$ away from each other to avoid overlap of $100-\mathrm{m}$ point count radii around each station. Eight stations were established at all sites except PRC, which was too small; only seven stations were established at this site. Stations were then classified as either being in conservation easement or developed areas.

All songbirds seen or heard within a 100-m radius of each sampling station were recorded using the dependent observer method. This method uses survey teams that consist of a primary observer who identified and quantified bird species and abundance, and a second observer who recorded data and helped identify and count any individuals missed by the primary observer (Nichols et al., 2000; Forcey, Anderson, Ammer, \& Whitmore, 2006). Two teams of two people conducted all of the bird sampling during this study. Songbirds were sampled once from every station at each site between May 19 and June 22, 2009. At each station, the sampling effort lasted 10 minutes, and all sampling occurred between 0500 and 0900 each day when weather conditions were favorable (e.g., no rain, light winds).

\subsection{Statistical Analyses}

Pre-treatment data handling varied depending on the analysis performed and is summarized here for clarity. All community metrics used in regression analyses were calculated using raw bird abundance data. However, abundances at the level of individual species and bird guilds were standardized (for each sample, by subtracting the sample mean from each data point and then dividing by the standard deviation) prior to regression analysis to account for differences in abundance that might occur between field survey teams. Similarly, all data were standardized prior to all multivariate PRIMER analyses (see below). However, PRIMER standardizes data by dividing raw abundance data in a sample by the total abundance for that sample, resulting in relative abundance data. As recommended for biological community data, this was done to address factors that might affect total abundance counts, such as differences in sample size or among survey teams (Clarke \& Gorley, 2006).

We used multidimensional scaling (MDS), analysis of similarity (ANOSIM) and similarity percentages (SIMPER) to compare bird communities between stations located in conservation easements and stations located in developed areas. This was done under the assumption that if there is no value in protecting land within cluster subdivisions then there will be no difference in bird communities between the two groups of stations. MDS was used to visualize patterns of similarity among stations in two-dimensional space, while ANOSIM (two-way nested model with station type nested within sites) was used to statistically compare bird communities between the two types of stations. SIMPER was used to identify the species that typify both groups of stations and species that most contribute to any dissimilarity between the station groups. Only data from the nine cluster subdivisions were used in these analyses; data from the COC and MET sites were not used here. Prior to each of these analyses, raw data were standardized as described above and square-root transformed to give less weight to common and ubiquitous species. Each of these analyses was conducted using PRIMER version 6.1.2 (Clarke \& Gorley, 2006).

We used a series of regression analyses to determine the proportion of protected land within cluster subdivisions that maximizes songbird diversity. We included the conventional subdivision and the undeveloped state forest as endpoints around the nine cluster subdivisions to examine patterns along a broader gradient of land protection. Because guidance for cluster and conservation subdivisions (the more refined successor to cluster subdivisions) in Rhode Island and elsewhere are often based on a percentage of the land being set aside using conservation easements, we used best-fit linear and nonlinear regression analyses to relate bird metrics at three scales (individual species, guilds, and communities) against cluster subdivision \%CE. At the community level, bird species richness and diversity were calculated at each site using raw abundance data and the Margalef and Shannon-Weiner indices, respectively. Mean standardized abundances of species in migratory (short-distance, permanent resident, or neotropical), human-tolerance (tolerant or intolerant), and habitat (forest, edge, or non-forest) guilds were calculated for each site and related to \%CE. Mean standardized abundances of individual species were also calculated for each site and related to \%CE. Regressions were run for all species that contributed to $90 \%$ of total bird abundance (Table 2). All regression analyses were conducted in SigmaPlot version 12 and SigmaStat version 3.5 software packages. 
Table 2. Summary of abundance metrics and guild affiliations for all bird species observed during this study

\begin{tabular}{|c|c|c|c|c|c|c|c|c|}
\hline \multirow[b]{2}{*}{ Species } & \multirow[b]{2}{*}{ Common name } & \multirow[b]{2}{*}{$\begin{array}{l}\text { Alpha } \\
\text { code }\end{array}$} & \multirow[b]{2}{*}{$\begin{array}{c}\text { Total \# } \\
\text { observed }\end{array}$} & \multirow[b]{2}{*}{$\begin{array}{c}\text { Relative } \\
\text { abundance } \\
(\%)\end{array}$} & \multirow[b]{2}{*}{$\begin{array}{c}\text { Cumulative } \\
(\%)\end{array}$} & \multicolumn{3}{|c|}{ Guilds } \\
\hline & & & & & & Migration & Tolerance & Habitat \\
\hline Turdus migratorius & American Robin & AMRO & 232 & 17.4 & 17.4 & SD & tolerant & edge \\
\hline Baeolophus bicolor & $\begin{array}{l}\text { Tufted } \\
\text { Titmouse }\end{array}$ & TUTI & 116 & 8.7 & 26.1 & PR & tolerant & edge \\
\hline Quiscalus quiscula & $\begin{array}{l}\text { Common } \\
\text { Grackle }\end{array}$ & COGR & 105 & 7.9 & 33.9 & SD & tolerant & non-forest \\
\hline $\begin{array}{l}\text { Dumetella } \\
\text { carolinensis }\end{array}$ & Gray Catbird & GRCA & 91 & 6.8 & 40.7 & NT & tolerant & edge \\
\hline $\begin{array}{l}\text { Cardinalis } \\
\text { cardinalis }\end{array}$ & $\begin{array}{l}\text { Northern } \\
\text { Cardinal }\end{array}$ & NOCA & 68 & 5.1 & 45.8 & PR & tolerant & edge \\
\hline Cyanocitta cristata & Blue Jay & BLJA & 56 & 4.2 & 50.0 & SD & tolerant & edge \\
\hline Spinus tristis & $\begin{array}{l}\text { American } \\
\text { Goldfinch }\end{array}$ & AMGO & 49 & 3.7 & 53.7 & SD & tolerant & edge \\
\hline Melospiza melodia & Song Sparrow & SOSP & 40 & 3.0 & 56.7 & SD & tolerant & non-forest \\
\hline Poecile atricapillus & $\begin{array}{l}\text { Black-capped } \\
\text { Chickadee }\end{array}$ & $\mathrm{BCCH}$ & 39 & 2.9 & 59.6 & PR & tolerant & edge \\
\hline Troglodytes aedon & House Wren & HOWR & 39 & 2.9 & 62.5 & $\mathrm{NT} / \mathrm{SD}$ & tolerant & edge \\
\hline Spizella passerine & $\begin{array}{l}\text { Chipping } \\
\text { Sparrow }\end{array}$ & CHSP & 36 & 2.7 & 65.2 & NT & tolerant & edge \\
\hline $\begin{array}{c}\text { Pipilo } \\
\text { erythrophthalmus }\end{array}$ & Eastern Towhee & EATO & 33 & 2.5 & 67.7 & NT & intolerant & forest/edge \\
\hline Zenaida macroura & Mourning Dove & MODO & 33 & 2.5 & 70.2 & SD & tolerant & non-forest \\
\hline $\begin{array}{l}\text { Bombycilla } \\
\text { cedrorum }\end{array}$ & Cedar Waxwing & CEDW & 31 & 2.3 & 72.5 & SD & intolerant & edge \\
\hline $\begin{array}{c}\text { Agelaius } \\
\text { phoeniceus }\end{array}$ & $\begin{array}{l}\text { Red-winged } \\
\text { Blackbird }\end{array}$ & RWBL & 31 & 2.3 & 74.8 & $\mathrm{SD} / \mathrm{NT}$ & tolerant & non-forest \\
\hline Seiurus aurocapilla & Ovenbird & OVEN & 27 & 2.0 & 76.9 & NT & intolerant & forest \\
\hline $\begin{array}{l}\text { Melanerpes } \\
\text { carolinus }\end{array}$ & $\begin{array}{l}\text { Red-bellied } \\
\text { Woodpecker }\end{array}$ & RBWO & 27 & 2.0 & 78.9 & PR & tolerant & edge \\
\hline $\begin{array}{c}\text { Corvus } \\
\text { brachyrhynchos }\end{array}$ & American Crow & AMCR & 23 & 1.7 & 80.6 & $\mathrm{SD}$ & tolerant & edge \\
\hline Aves & $\begin{array}{l}\text { Unidentified } \\
\text { Bird }\end{array}$ & UNBI & 23 & 1.7 & 82.3 & & & \\
\hline Molothrus ater & $\begin{array}{l}\text { Brown-headed } \\
\text { Cowbird }\end{array}$ & $\mathrm{BHCO}$ & 20 & 1.5 & 83.8 & SD & intolerant & non-forest \\
\hline $\begin{array}{l}\text { Catharus } \\
\text { fuscescens }\end{array}$ & Veery & VEER & 18 & 1.3 & 85.2 & NT & intolerant & forest \\
\hline Vireo olivaceus & Red-eyed Vireo & REVI & 17 & 1.3 & 86.4 & NT & intolerant & forest \\
\hline Sitta carolinensis & $\begin{array}{l}\text { White-breasted } \\
\text { Nuthatch }\end{array}$ & WBNU & 16 & 1.2 & 87.6 & PR & intolerant & edge \\
\hline Setophaga petechia & Yellow Warbler & YEWA & 16 & 1.2 & 88.8 & NT & tolerant & edge \\
\hline Passer domesticus & House Sparrow & HOSP & 14 & 1.0 & 89.9 & SD & tolerant & non-forest \\
\hline $\begin{array}{l}\text { Thryothorus } \\
\text { ludovicianus }\end{array}$ & Carolina Wren & CARW & 13 & 1.0 & 90.9 & PR & tolerant & edge \\
\hline Setophaga pinus & Pine Warbler & PIWA & 13 & 1.0 & & SD & intolerant & forest \\
\hline Picoides pubescens & $\begin{array}{c}\text { Downy } \\
\text { Woodpecker }\end{array}$ & DOWO & 12 & 0.9 & & PR & tolerant & edge \\
\hline Geothlypis trichas & $\begin{array}{l}\text { Common } \\
\text { Yellowthroat }\end{array}$ & COYE & 11 & 0.8 & & NT & intolerant & edge \\
\hline Contopus virens & $\begin{array}{c}\text { Eastern } \\
\text { Wood-Pewee }\end{array}$ & EAPW & 11 & 0.8 & & NT & intolerant & forest/edge \\
\hline Myiarchus crinitus & $\begin{array}{l}\text { Great Crested } \\
\text { Flycatcher }\end{array}$ & GCFL & 11 & 0.8 & & NT & tolerant & edge \\
\hline $\begin{array}{l}\text { Hylocichla } \\
\text { mustelina }\end{array}$ & Wood Thrush & WOTH & 10 & 0.7 & & NT & intolerant & forest \\
\hline
\end{tabular}


Table 2. Summary of abundance metrics and guild affiliations for all bird species observed during this study (continued)

\begin{tabular}{|c|c|c|c|c|c|c|c|c|}
\hline \multirow[b]{2}{*}{ Species } & \multirow[b]{2}{*}{ Common name } & \multirow[b]{2}{*}{$\begin{array}{l}\text { Alpha } \\
\text { code }\end{array}$} & \multirow[b]{2}{*}{$\begin{array}{c}\text { Total \# } \\
\text { observed }\end{array}$} & \multirow[b]{2}{*}{$\begin{array}{c}\text { Relative } \\
\text { abundance } \\
(\%)\end{array}$} & \multirow[b]{2}{*}{$\begin{array}{c}\text { Cumulative } \\
(\%)\end{array}$} & \multicolumn{3}{|c|}{ Guilds } \\
\hline & & & & & & Migration & Tolerance & Habitat \\
\hline $\begin{array}{l}\text { Carpodacus } \\
\text { mexicanus }\end{array}$ & House Finch & HOFI & 6 & 0.4 & & SD & tolerant & edge \\
\hline Sayornis phoebe & Eastern Phoebe & EAPH & 5 & 0.4 & & SD & tolerant & non-forest \\
\hline Spizella pusilla & Field Sparrow & FISP & 5 & 0.4 & & SD & intolerant & edge \\
\hline Mniotilta varia & $\begin{array}{c}\text { Black-and-white } \\
\text { Warbler }\end{array}$ & BAWW & 4 & 0.3 & & NT & intolerant & forest \\
\hline Picoides villosus & $\begin{array}{c}\text { Hairy } \\
\text { Woodpecker }\end{array}$ & HAWO & 4 & 0.3 & & PR & tolerant & edge \\
\hline Colaptes auratus & $\begin{array}{l}\text { Northern } \\
\text { Flicker }\end{array}$ & NOFL & 4 & 0.3 & & SD & intolerant & edge \\
\hline Mimus polyglottos & $\begin{array}{c}\text { Northern } \\
\text { Mockingbird }\end{array}$ & NOMO & 4 & 0.3 & & PR & tolerant & non-forest \\
\hline Setophaga discolor & Prairie Warbler & PRAW & 4 & 0.3 & & NT & intolerant & edge \\
\hline $\begin{array}{l}\text { Archilochus } \\
\text { colubris }\end{array}$ & $\begin{array}{l}\text { Ruby-throated } \\
\text { Hummingbird }\end{array}$ & RTHU & 3 & 0.2 & & NT & tolerant & edge \\
\hline Setophaga ruticilla & $\begin{array}{c}\text { American } \\
\text { Redstart }\end{array}$ & AMRE & 2 & 0.1 & & NT & intolerant & edge \\
\hline Sturnus vulgaris & $\begin{array}{c}\text { European } \\
\text { Starling }\end{array}$ & EUST & 2 & 0.1 & & $\mathrm{SD}$ & tolerant & non-forest \\
\hline $\begin{array}{l}\text { Tachycineta } \\
\text { bicolor }\end{array}$ & Tree Swallow & TRES & 2 & 0.1 & & NT & intolerant & non-forest \\
\hline Parulidae & $\begin{array}{l}\text { Unidentified } \\
\text { Warbler }\end{array}$ & UNWA & 2 & 0.1 & & & & \\
\hline Empidonax traillii & $\begin{array}{l}\text { Willow } \\
\text { Flycatcher }\end{array}$ & WIFL & 2 & 0.1 & & NT & intolerant & edge \\
\hline $\begin{array}{l}\text { Vermivora } \\
\text { cyanoptera }\end{array}$ & $\begin{array}{l}\text { Blue-winged } \\
\text { Warbler }\end{array}$ & BWWA & 1 & 0.1 & & NT & intolerant & edge \\
\hline $\begin{array}{l}\text { Pheucticus } \\
\text { ludovicianus }\end{array}$ & $\begin{array}{c}\text { Rose-breasted } \\
\text { Grosbeak }\end{array}$ & RBGR & 1 & 0.1 & & NT & intolerant & edge \\
\hline Piranga olivacea & Scarlet Tanager & SCTA & 1 & 0.1 & & NT & intolerant & forest \\
\hline Emberizidae & $\begin{array}{l}\text { Unidentified } \\
\text { Sparrow }\end{array}$ & UNSP & 1 & 0.1 & & & & \\
\hline $\begin{array}{c}\text { Setophaga } \\
\text { coronata }\end{array}$ & $\begin{array}{l}\text { Yellow-rumped } \\
\text { Warbler }\end{array}$ & YRWA & 1 & 0.1 & & NT & intolerant & forest \\
\hline
\end{tabular}

Note: Alpha codes were derived from Pyle and DeSante (2011). Guild information was compiled from Ehrlich, Dobkin, \& Wheye (1988), Lussier et al. (2006), Pidgeon et al. (2007), and personal experience.

Finally, we used ARCGIS to calculate the mean distance that each species (and select guilds) was found from the nearest development edge to provide further insight into species' responses to cluster subdivision design. For each species, these distances were calculated by multiplying the number of individuals found at each station by the distance from the center of that station to the nearest development edge, and then summing these across all stations and dividing by the total abundance of that species.

\section{Results}

\subsection{Bird Community Composition}

Forty-eight songbird species (not including birds classified as unidentified, unidentified sparrows or unidentified warblers) and 1335 individuals were recorded during this study. Twenty-six species comprised over $90 \%$ of all observations (Table 2). The American Robin was by far the most abundant species (it comprised $17 \%$ of the entire community), followed by Tufted Titmouse (9\%), Common Grackle (8\%), Gray Catbird (7\%), and Northern Cardinal (5\%). In contrast, 24 species each comprised less than $1 \%$ of the entire songbird community. At the guild level, the bird community was dominated by short-distance migrants ( $47 \%$ of the migratory guild), 
neotropical migrants $(23 \%)$ and permanent residents $(22 \%)$. It was dominated by edge species $(71 \%$ of the habitat guild), followed by non-forest (19\%), forest-interior (7\%), and forest/edge habitat (3\%) species. Human tolerant species were far more abundant ( $81 \%$ of the community) than were human-intolerant species (18\%).

\subsection{Conservation Easement Versus Developed Stations}

An MDS plot of all stations from the nine cluster subdivisions shows that the conservation easement stations and the developed stations generally grouped apart from each other, but that there was some overlap; a number of conservation easement stations clearly intermix with the developed stations (Figure 3). A statistically significant difference was found between bird communities at stations protected by conservation easements and those that were developed (ANOSIM; global $\mathrm{R}=0.32 ; \mathrm{p}=0.001$ ). Based on SIMPER, 30 species contributed to over $90 \%$ of the dissimilarity between the two station groups (Table 3), but most of these species were ubiquitously found through the study area (e.g., Tufted Titmouse, Northern Cardinal, American Robin and Gray Catbird). Many of these same species also typified both conservation easement and developed stations, but some species were only identified by SIMPER as typifying one set of stations or the other. Species that only typified conservation easement stations include Eastern Towhee (contributed $4.4 \%$ to overall similarity of easement stations), Black-capped Chickadee (2.4\%), Ovenbird (2.4\%), Red-winged Blackbird (1.8\%), White-breasted Nuthatch (1.7\%), and Red-eyed Vireo (1.2\%) (Table 3). In contrast, species that only typified developed stations include the Common Grackle (contributed $12.9 \%$ to overall similarity of these stations), Song Sparrow (7.0\%), American Goldfinch (5.2\%), and Brown-headed Cowbird (1.7\%).

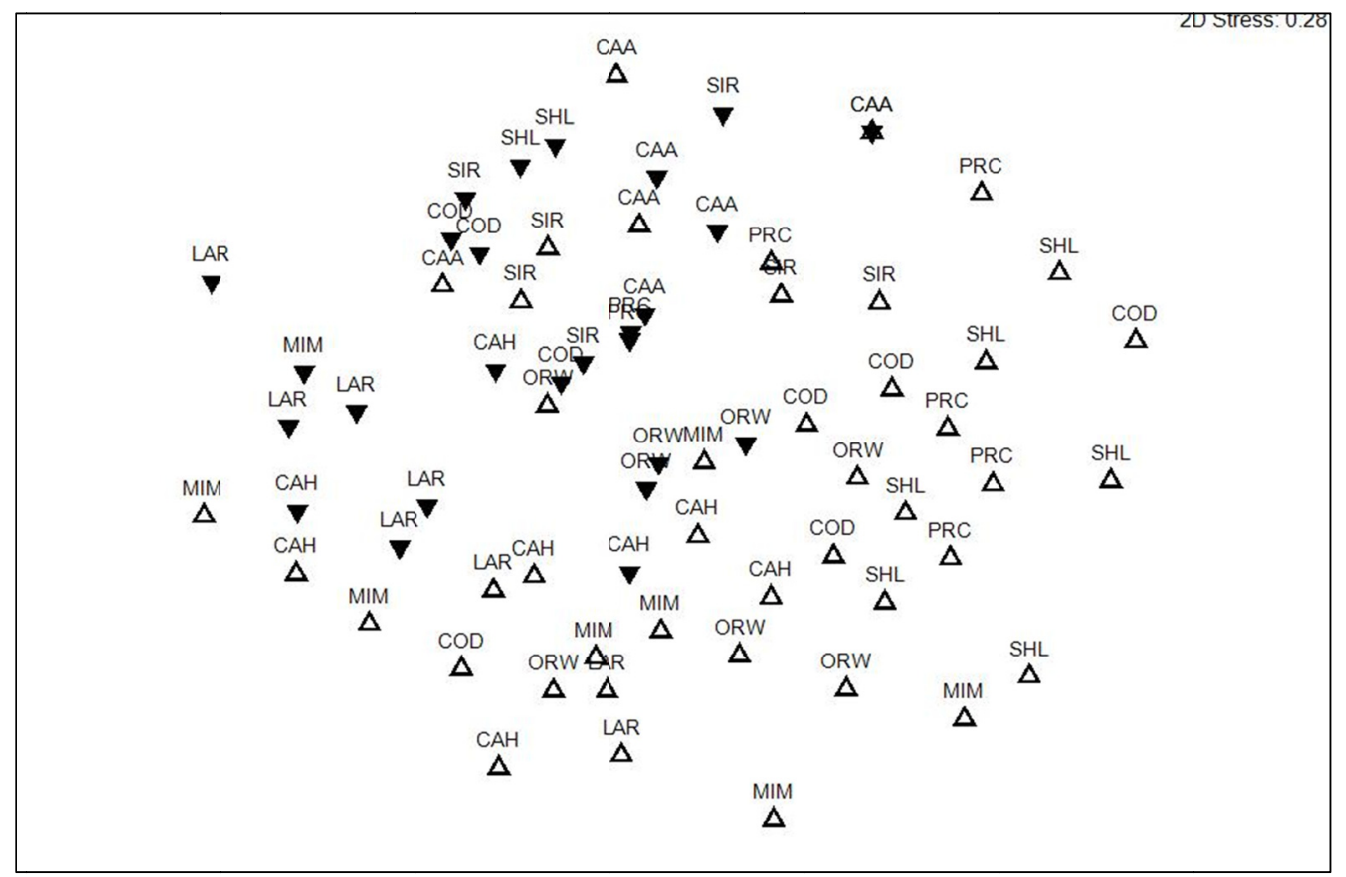

Figure 3. Two-dimensional multidimensional scaling (MDS) plot of individual bird survey stations

Note: Each station is labeled with its three-letter site code. Symbols indicate whether the station is within a conservation easement $(\triangle)$ or a developed $(\nabla)$ area 
Table 3. Results from SIMPER analyses comparing conservation easement (CE) and developed (Dev) stations

\begin{tabular}{|c|c|c|c|c|c|}
\hline \multirow{2}{*}{ Species code } & \multicolumn{2}{|c|}{ Rel. Abun. } & \multicolumn{3}{|c|}{$\%$ Contribution } \\
\hline & $\mathrm{CE}$ & Dev & CE similarity & Dev similarity & $\mathrm{CE} / \mathrm{Dev}$ dissimilarity \\
\hline COGR & 0.54 & 2.66 & & 12.91 & 6.70 \\
\hline TUTI & 2.73 & 1.98 & 16.17 & 8.31 & 5.93 \\
\hline NOCA & 1.86 & 1.49 & 9.02 & 4.91 & 4.91 \\
\hline AMRO & 3.75 & 4.25 & 30.25 & 29.32 & 4.85 \\
\hline GRCA & 2.24 & 2.19 & 13.63 & 11.62 & 4.71 \\
\hline AMGO & 0.58 & 1.67 & & 5.16 & 4.46 \\
\hline SOSP & 0.44 & 1.59 & & 6.99 & 4.10 \\
\hline BLJA & 1.24 & 1.01 & 4.26 & 1.99 & 4.06 \\
\hline CHSP & 0.56 & 1.43 & 0.98 & 4.80 & 3.65 \\
\hline EATO & 1.26 & 0.45 & 4.42 & & 3.54 \\
\hline $\mathrm{BCCH}$ & 0.94 & 0.87 & 2.39 & & 3.37 \\
\hline RBWO & 0.71 & 0.85 & 1.43 & 2.00 & 2.83 \\
\hline RWBB & 0.98 & 0.24 & 1.75 & & 2.82 \\
\hline UNBI & 0.24 & 0.95 & & & 2.82 \\
\hline HOWR & 0.46 & 0.89 & & & 2.82 \\
\hline MODO & 0.53 & 0.80 & 0.87 & 1.75 & 2.70 \\
\hline OVEN & 0.91 & 0.14 & 2.37 & & 2.51 \\
\hline $\mathrm{BHCO}$ & 0.17 & 0.93 & & 1.72 & 2.50 \\
\hline AMCR & 0.45 & 0.75 & & & 2.41 \\
\hline WBNU & 0.75 & 0.23 & 1.67 & & 2.30 \\
\hline REVI & 0.68 & 0.29 & 1.23 & & 2.17 \\
\hline YEWA & 0.49 & 0.42 & & & 2.03 \\
\hline CEWA & 0.36 & 0.48 & & & 1.92 \\
\hline CAWR & 0.50 & 0.18 & & & 1.56 \\
\hline COYE & 0.49 & 0.14 & & & 1.47 \\
\hline VEER & 0.54 & 0.08 & & & 1.47 \\
\hline HOSP & 0.07 & 0.51 & & & 1.47 \\
\hline DOWO & 0.37 & 0.25 & & & 1.40 \\
\hline EWPE & 0.47 & 0.08 & & & 1.31 \\
\hline WOTH & 0.48 & 0.00 & & & 1.26 \\
\hline
\end{tabular}

Note: Abundance is a relative number and was calculated using standardized and square-root transformed data in PRIMER. For each species, when applicable, its percent contribution to bird community similarity within CE stations, similarity within Dev stations, and dissimilarity between CE and Dev stations is shown.

\subsection{Bird Relationships With Subdivision \%CE}

Eight bird species exhibited statistically significant relationships with \%CE (Table 4). Abundances of American Robin, Common Grackle, House Wren, Mourning Dove, and Song Sparrow all declined linearly with increasing \%CE. Abundances of American Goldfinch and Black-capped Chickadee peaked at 70 and $63 \% \mathrm{CE}$, respectively, and Ovenbird abundance increased exponentially with increasing \%CE. Similarly, five bird guilds were statistically related to \%CE. Short-distance migrants, human tolerant birds, and non-forest habitat birds all decreased linearly with increasing \%CE, while human intolerant birds increased linearly and forest interior birds increased exponentially with increasing \%CE (Table 4). At the bird community level, species richness and diversity both exhibited statistically significant nonlinear relationships with $\% \mathrm{CE}$; distinct peaks were found for 
richness at $73 \% \mathrm{CE}$ and for diversity at $74 \% \mathrm{CE}$ (Table 4; Figure 4). No significant relationship were found for any other species or bird guilds that were examined, or for total bird abundance ( $p>0.05$ in each case).

Table 4. A summary of significant relationships found between bird species, guild, and community variables and $\% \mathrm{CE}$ using linear and nonlinear regression analyses

\begin{tabular}{|c|c|c|c|c|c|c|}
\hline & Independent Variable & Model type & Trend & $\mathrm{F}$ & $\mathrm{P}$ & $\mathrm{R}^{2}$ \\
\hline \multirow[t]{8}{*}{ Species } & AMGO & Peak & Peak at $70 \% \mathrm{CL}$ & 67.04 & $<0.0001$ & 0.93 \\
\hline & AMRO & Linear & Decrease & 5.23 & 0.048 & 0.37 \\
\hline & $\mathrm{BCCH}$ & Peak & Peak at $63 \% \mathrm{CE}$ & 5.91 & 0.027 & 0.60 \\
\hline & COGR & Linear & Decrease & 36.65 & 0.000 & 0.78 \\
\hline & HOWR & Linear & Decrease & 5.63 & 0.042 & 0.38 \\
\hline & MODO & Linear & Decrease & 8.51 & 0.017 & 0.49 \\
\hline & OVEN & Exponential & Increase & 11.83 & 0.004 & 0.75 \\
\hline & SOSP & Linear & Decrease & 31.59 & 0.000 & 0.78 \\
\hline \multirow[t]{5}{*}{ Guild } & Short-distance migrant & Linear & Decrease & 15.42 & 0.004 & 0.63 \\
\hline & Human tolerant & Linear & Decrease & 8.52 & 0.017 & 0.49 \\
\hline & Human intolerant & Linear & Increase & 10.84 & 0.009 & 0.55 \\
\hline & Forest interior & Exponential & Increase & 18.41 & 0.002 & 0.67 \\
\hline & Non-forest & Linear & Decrease & 29.52 & $<0.001$ & 0.77 \\
\hline \multirow[t]{2}{*}{ Community } & Species diversity & Peak & Peak at $74 \% \mathrm{CE}$ & 10.57 & 0.005 & 0.82 \\
\hline & Species richness & Peak & Peak at $73 \% \mathrm{CE}$ & 16.45 & 0.002 & 0.88 \\
\hline
\end{tabular}

A.

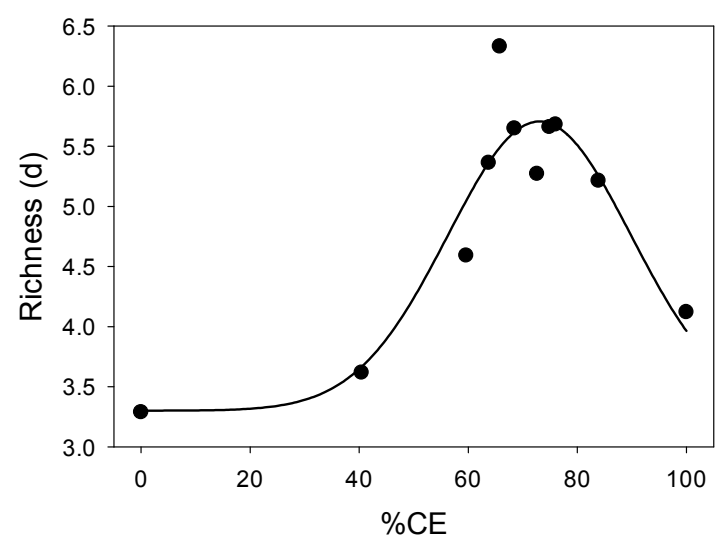

B.

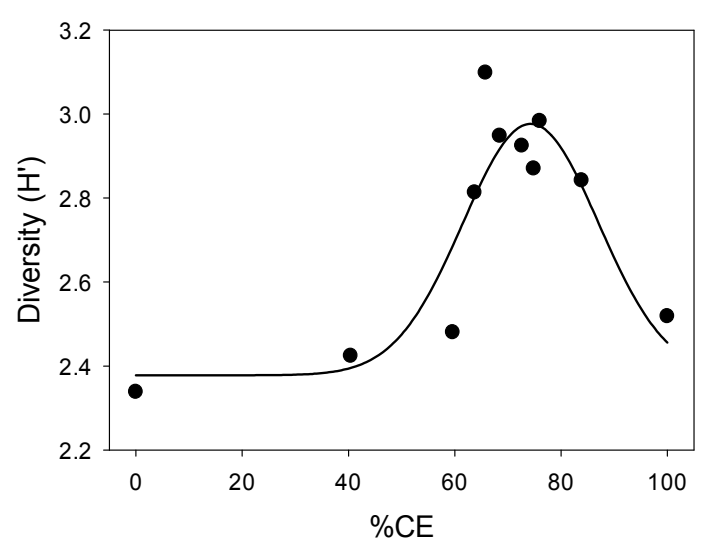

Figure 4. Relationships between Margalef species richness (A) and Shannon-Weiner diversity (B) and cluster subdivision \%CE

Note: Both indices were significantly related to $\% \mathrm{CE}$ based on non-linear regression; richness peaked at $73 \% \mathrm{CE}$; diversity peaked at $74 \% \mathrm{CE}$

\subsection{Distance to Development Edges}

At the habitat guild level, forest interior species were found at a mean distance of $148 \mathrm{~m}$ from the nearest development edge. In contrast, human-associated, non-forest species were found in close proximity to development edges (mean of $15 \mathrm{~m}$ ); edge species were predictably found at intermediate distances (mean of 49 $\mathrm{m})$. Seven of the ten species found at the greatest mean distance from a development edge were forest interior species (ranging from Red-eyed Vireo at $117 \mathrm{~m}$ to Pine Warbler at $222 \mathrm{~m}$; Figure 5). 


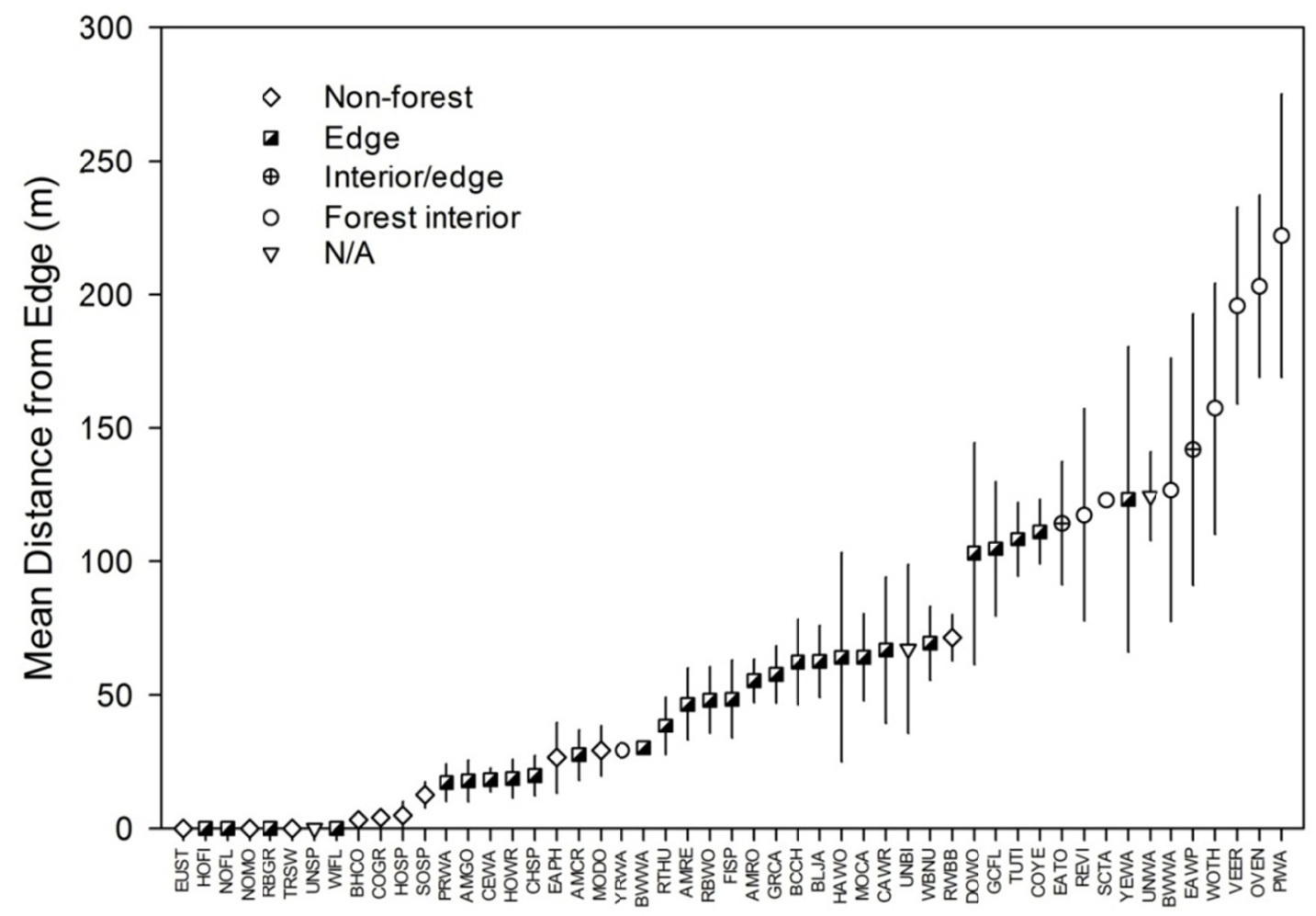

Figure 5. Mean distance each bird species was found from the nearest residential development edge. Birds are symbolized based on habitat guild; error bars represent $\pm 1 \mathrm{SE}$

\section{Discussion}

Debate between planners and natural resource managers continues (at least in Rhode Island) in regards to the value of cluster subdivisions for wildlife. This is likely facilitated by a shortage of published studies addressing this issue. Our study helps fill this gap and provides evidence that well-designed cluster subdivisions can provide valuable habitat for songbird communities and bird species of concern. Specifically, we found significant differences in songbird community composition between developed and protected areas within cluster subdivisions. This difference, and the fact that it was largely due to the retention of some forest interior species in conservation easement areas, demonstrates that protected habitat within cluster developments can provide value for these species and are not completely subsumed by nearby developed areas. Conversely, Lenth et al. (2006) found that bird community composition and the number of bird nests in cluster subdivisions were more similar to conventional dispersed developments than undeveloped areas. The contrasting results from these two studies suggest that the value of cluster subdivisions for songbirds may not be universal and instead may depend on geographic region, dominant local habitat types, and study design and methods. Unfortunately, these are the only studies to date that have directly quantified bird use of cluster subdivisions and more case studies are clearly needed.

Our study shows that conservation easement areas within cluster subdivisions enhance songbird diversity, yet are clearly impacted by proximate and/or regional human development. Ubiquitous suburban species such as American Robin, Tufted Titmouse, Northern Cardinal, Gray Catbird, and Blue Jay were dominant throughout most study sites regardless of habitat. This is another clear example of biotic homogenization, which is a well-documented phenomenon where native species become replaced by a small number of ubiquitous, abundant, and synanthropic species (McKinney, 2006; Devictor, Julliard, Couvet, Lee, \& Jiguet, 2007; van Rensburg, Peacock, \& Robertson, 2009). Despite this, our SIMPER analyses show that bird communities in conservation easement areas were still typified and defined in part by less abundant, forest affiliated species such as Eastern Towhee, Ovenbird, and Red-eyed Vireo. This agrees with van Rensburg et al. (2009) who found that suburban areas in South Africa provided important habitat for native bird species even though communities were also dominated by a ubiquitous non-native species (Common Myna Acridotheres tristis). In cases such as these, it may be necessary to use analytical techniques (e.g., transformations to downweight dominant species were used 
here) that dampen the potentially masking effects of dominant species in order to elucidate responses by less abundant species that may be of conservation concern.

In our study, songbird occurrence in protected land within cluster subdivisions depended on the bird metrics that were used and the relative amount of land that was protected. This in turn shows that determining the appropriate amount of land to protect depends on a priori conservation goals. For example, if the goal is to maximize songbird biodiversity, then approximately $70-75 \%$ of the land should be protected. This finding is an example of intermediate levels of disturbance leading to enhanced biodiversity, which occurs when the influx of new species into a community from disturbance (e.g., residential development) is faster than the loss of disturbance-sensitive species (Blair, 1996; McKinney, 2002; Crooks, Suarez, \& Bolger, 2004; McKinney, 2006). In our study, biodiversity began to decrease when development exceeded approximately $25-30 \%$ of the parcel. However, one issue with using enhanced local biodiversity as a conservation goal is that richness and diversity indices are both indiscriminate; they incorporate synanthropic, urban adaptable species that benefit from expanding residential development and that are not generally of conservation concern.

Conversely, an even higher percentage of land should be protected within subdivisions if human intolerant, forest interior birds (e.g., Ovenbird, Pine Warbler, and Veery) are of primary concern. Bird species in these guilds are negatively impacted by increasing residential development (Kluza, Griffin, \& DeGraaf, 2000), are generally declining (Robbins, Sauer, Greenberg, \& Droege, 1989; Jones, McCann, \& McConville, 2001; Blodget, Dettmers, \& Scanlon, 2009), and may be a more appropriate focus of conservation efforts associated with cluster subdivisions than songbird biodiversity (Lenth et al., 2006). Our data show that abundances of birds classified within human intolerant and forest interior guilds increase linearly and exponentially with increasing \%CE, respectively. Unfortunately, it is difficult to quantify or recommend a specific relative or absolute amount of land that should be protected for these species. One issue is that the amount of required land is species-specific (Robbins et al., 1989), but estimates also vary among regions and studies. For example, a threshold of $12 \%$ residential development was quantified for human-intolerant species in Rhode Island riparian habitats by Lussier, Enser, Dasilva and Charpentier (2006). In other regions, Donnelly and Marzluff (2004) found that most forest interior species required at least 42 ha of protected habitat near Seattle, WA and Fernández-Juricic (2004) determined that forest specialists needed 20-90 ha of protected habitat in Madrid, Spain, depending on species.

If the preservation of forest interior species is a conservation goal, then protected land within cluster subdivisions should be placed into large contiguous blocks that maximize the amount of forest away from the influence of residential development edges. Our data show that forest interior birds were found at a mean distance of $148 \mathrm{~m}$ away from the nearest residential development edge, but edge effects vary by species and among studies. For example, Jones et al. (2001) define forest interior as $300 \mathrm{ft}(91 \mathrm{~m})$ away from adjacent habitats such as residential development, while Mason et al. (2007) found some forest interior bird species only when greenways were wider than $100 \mathrm{~m}$; other species (e.g., Ovenbird) were only found when greenways were at least $300 \mathrm{~m}$ wide. The required distance away from development edges for forest-interior species is clearly variable and species-specific. Further, even if most of the land is protected within a well-designed cluster subdivision in Rhode Island, it will be difficult to protect forest-interior habitats because parcel sizes are relatively small (the mean size was 34 ha among our study sites). This emphasizes that it is critical to consider all existing and potential future habitat types that exist in abutting parcels in the surrounding matrix to try to create the largest possible blocks of habitat across multiple protected parcels (i.e., greenways). This further highlights the need to move beyond standard cluster subdivisions and towards conservation subdivisions, which have stricter land preservation requirements and a higher potential for creating open space greenways by connecting multiple protected parcels (Flinker, 2003; Arendt, 2004; Freeman \& Bell, 2011).

The results of our study should be interpreted carefully because the use of only structural data such as abundance or density can be misleading (Van Horn, 1983). For example, Porneluzi, Bednarz, Goodrich, Zawada, \& Hoover (1993) found that Ovenbird abundance in large (> 100 ha) forest fragments in eastern Pennsylvania were similar to a larger unfragmented forest, but fragments of approximately 180 ha were still not big enough to support successfully breeding Ovenbirds based on nesting success (a functional measure). In their study, the smaller protected habitats were serving as sinks for individuals or breeding pairs emigrating from nearby larger source forests. Ovenbirds and other forest-interior species were generally common in conservation easement areas in our study, but it is possible that these areas were also merely sinks for non-breeding birds that emigrated from much larger protected forests in nearby western Rhode Island. Unfortunately, information on the functional responses of songbirds to the protection of habitats within cluster subdivisions in the eastern United States does not exist. Without these data, land protection efforts associated with cluster subdivisions must rely on the best available structural assessments to determine how much land needs to be protected to achieve the desired conservation goals. 
The results of our study should be interpreted carefully for a few additional reasons. First, our study was purposefully conducted only in a suburban landscape/matrix and the results may differ if the setting was either more urbanized or more rural or exurban. Many studies clearly show that the surrounding matrix can have a strong effect on bird habitat use (reviewed by Prevedello \& Vieira, 2010) and a study such as ours should be conducted in matrix settings that are both more urbanized and more natural to provide a better understanding of how songbirds respond to cluster subdivisions in Rhode Island and southern New England. Second, our results may not apply across different dominant habitat types. In our study, forest comprised $90 \%$ of the land protected within cluster subdivisions. In other areas of the United States, however, cluster subdivisions are built on land that is dominated by farmland, ranchland, prairie, and other, non-forested habitat types (Brabec, 2001; Lenth et al., 2006). While some of our results may translate across habitats, the specific responses of birds to land protection within cluster subdivisions should be evaluated across a variety of different habitats. Finally, we utilized a gradient approach by including data from one conventional development and one state forest. A follow-up study could build upon this by quantifying birds from replicated conventional development and protected forest sites. This would produce a database of songbird metrics from large protected southern New England forests that in turn could provide quantifiable reference targets to help assess the effectiveness of future land preservation efforts in cluster developments.

Based on our results, an ideal cluster or conservation development in suburban Rhode Island would protect at least $70-75 \%$ of the original undeveloped parcel of land, and it would maximize the amount of forest away from residential development edges to benefit bird community metrics and forest interior species (our data suggest a conservative, minimum distance of approximately $150 \mathrm{~m}$ ). Based on these recommendations, a site such as Misty Meadows provides a good example to follow. It protects $84 \%$ of the entire parcel of land as open space, has a low edge density, and most of the protected land lies within one large contiguous block, $33 \%$ of which is at least $150 \mathrm{~m}$ from the nearest development edge (although this would drop to only $10 \%$ if two abutting parcels that are currently unprotected were to be developed; this again illustrates the need to consider both current and future land uses when designing cluster subdivisions). Its protected lands also directly abut an additional 65 ha of cluster subdivision protected land, thus creating a protected greenway of over 100 ha. At the same time, this site still contains 29 housing units with a median value that is $88 \%$ higher than the median value of the entire Town of North Kingstown (www.zillow.com; accessed April 2012). Thus, a well-designed cluster subdivision can develop a neighborhood of relatively valuable houses while simultaneously protecting large contiguous blocks of forested open space that minimize development edges and benefit sensitive songbirds.

\section{Conclusion}

In conclusion, our study shows that cluster subdivisions can enhance songbird biodiversity and provide habitat for species of concern, depending on the amount and configuration of the land that is protected. Currently, the State of Rhode Island does not recommend or require that cluster subdivisions protect a certain percentage of land. At the time of this writing, however, a bill had been introduced into the State legislature that would require the protection of a minimum of $50 \%$ of the buildable land within a parcel that is to be developed as a conservation subdivision. This measure of land protection differs from the percent of the entire parcel of land $(\% \mathrm{CE})$ that we used in our study, but our results are still relevant for planning purposes in Rhode Island. If it is accepted that at least $70-75 \%$ of the entire parcel should be protected, then the proposed conservation subdivision requirement is only adequate when a parcel is at least $50 \%$ unbuildable (e.g., due to wetland, groundwater, or slope issues, etc.); anything less than this and the proposed requirement will not necessarily protect the $70-75 \%$ target. To address this, language could be changed to require the protection of at least $50 \%$ of the buildable land or $75 \%$ of the entire parcel, whichever is greater. Regardless of how well a new cluster or conservation subdivision is designed, it will alter the composition of the songbird community that was present in the original undeveloped parcel of land. Results from our study can be used to help guide the planning and design of future subdivisions in suburban Rhode Island and elsewhere to help achieve desired wildlife conservation goals.

\section{Acknowledgements}

We would like to thank Jennifer West and Rose Cournoyer for their help with field data collections and Robin Weber for GIS support and for creating Figures 1 and 2. Jennifer West also helped us identify and refine the goals and design of the study. Jon Reiner and his staff from the Town of North Kingstown Planning Department also provided insight into the goals and design of the study as well as information on cluster subdivisions throughout the Town. We especially want to thank the homeowners and homeowner associations of the nine cluster subdivisions that were included in this study. Partial funding for this project was provided by NOAA grant \#NA08NOS4200373 to the Narragansett Bay National Estuarine Research Reserve. Mention of trade names or commercial products does not constitute endorsement or recommendation. Although the research described in this article has been funded wholly by the U.S. Environmental Protection Agency, it has not been 
subjected to Agency-level review. Therefore, it does not necessarily reflect the views of the Agency. This is the Office of Research and Development, National Health and Environmental Effects Research Laboratory, Atlantic Ecology Division contribution number AED-08-028.

\section{References}

Arendt, R. (1994). Rural by Design: Maintaining Small Town Character. Washington, DC: American Planning Association.

Arendt, R. (1996). Conservation Design for Subdivisions: A Practical Guide to Creating Open Space Networks. Washington, DC: Island Press.

Arendt, R. (2004). Linked landscapes: Creating greenway corridors through conservation subdivision strategies in the north eastern and central United States. Landscape and Urban Planning, 68, 241-269. http://dx.doi.org/10.1016/S0169-2046(03)00157-9

Blair, R. B. (1996). Land use and avian species diversity along an urban gradient. Ecological Applications, 6, 506-519. http://dx.doi.org/10.2307/2269387

Blodget, B. G., Dettmers, R., \& Scanlon, J. (2009). Status and trends of birds in an extensive western Massachusetts forest. Northeastern Naturalist, 16, 423-442. http://dx.doi.org/10.1656/045.016.n310

Brabec, E. (1994). The economics of preserving open space. In R. Arendt (Ed.), Rural by Design: Maintaining Small Town Character (pp. 71-89). Washington, DC: American Planning Association.

Brabec, E. (2001). An evaluation of the effectiveness of cluster development in the town of Southampton, New York. Urban Ecosystems, 4, 27-47. http://dx.doi.org/10.1023/A:1021825601746

Brown, D. G., Johnson, K. M., Loveland, T. R., \& Theobald, D. M. (2005). Rural land-use trends in the conterminous United States, 1950-2000. Ecological Applications, 15, 1851-1863. http://dx.doi.org/10.1890/03-5220

Chace, J. F., \& Walsh, J. J. (2006). Urban effects on native avifauna: a review. Landscape and Urban Planning, 74, 46-69. http://dx.doi.org/10.1016/j.landurbplan.2004.08.007

Clarke, K. R., \& Gorley, R. N. (2006). PRIMER v6: User Manual/Tutorial. Plymouth, United Kingdom: PRIMER-E ltd.

Crooks, K. R., Suarez, A. V., \& Bolger, D. T. (2004). Avian assemblages along a gradient of urbanization in a highly fragmented landscape. Biological Conservation, 115, 451-462. http://dx.doi.org/10.1016/S0006-3207(03)00162-9

Czech, B., Krausman, P. R., \& Devers, P. K. (2000). Economic associations among causes of species endangerment in the United States. Bioscience, 50, 593-601. http://dx.doi.org/10.1641/0006-3568(2000)050[0593:EAACOS]2.0.CO;2

Devictor, V., Julliard, R., Couvet, D., Lee, A., \& Jiguet, F. (2007). Functional homogenization effect of urbanization on bird communities. Conservation Biology, 21, 741-751. http://dx.doi.org/10.1111/j.1523-1739.2007.00671.x

Donnelly, R., \& Marzluff, J. M. (2004). Importance of reserve size and landscape context to urban bird conservation. Conservation Biology, 18, 733-745. http://dx.doi.org/10.1111/j.1523-1739.2004.00032.x

Drummond, M. A., \& Loveland, T. R. (2010). Land-use pressure and a transition to forest-cover loss in the eastern United States. Bioscience, 60, 286-298. http://dx.doi.org/10.1525/bio.2010.60.4.7

Duh, J., Shandas, V., Chang, H., \& George, L. A. (2008). Rates of urbanization and the resiliency of air and water quality. Science of the Total Environment, 400, 238-256. http://dx.doi.org/10.1016/j.scitotenv.2008.05.002

Ehrlich, P. R., Dobkin, D. S., \& Wheye, D. (1988). The Birder's Handbook: A Field Guide to the Natural History of North American Birds. New York: Simon and Schuster.

Fernández-Juricic, E. (2004). Spatial and temporal analysis of the distribution of forest specialists in an urban-fragmented landscape (Madrid, Spain). Implications for local and regional bird conservation. Landscape and Urban Planning, 69, 17-32. http://dx.doi.org/10.1016/j.landurbplan.2003.09.001

Flinker, P. (2003). The Rhode Island Conservation Development Manual. Providence, RI: Rhode Island Department of Environmental Management. 
Forcey, G. M., Anderson, J. T., Ammer, F. K., \& Whitmore, R. C. (2006). Comparison of two double observer point count approaches for estimating breeding bird abundance. Journal of Wildlife Management, 70, 1674-1681. http://dx.doi.org/10.2193/0022-541X(2006)70[1674:COTDPA]2.0.CO;2

Freeman, R. C., \& Bell, K. P. (2011). Conservation versus cluster subdivisions and implications for habitat $\begin{array}{lllll}\text { connectivity. } \quad \text { Landscape } \text { and } & \text { 30-42. }\end{array}$ http://dx.doi.org/10.1016/j.landurbplan.2010.12.019

Jones, C., McCann, J., \& McConville, S. (2001). A guide to the conservation of forest interior dwelling birds in the Chesapeake Bay critical area. Report from the Critical Area Commission for the Chesapeake and Atlantic Coastal Bays.

Kluza, D. A., Griffin, C. R., \& DeGraaf, R. M. (2000). Housing developments in rural New England: effect on forest birds. Animal Conservation, 3, 15-26. http://dx.doi.org/10.1017/S1367943000000706

Lenth, B. A., Knight, R. L., \& Gilgert, W. C. (2006). Conservation value of clustered housing developments. Conservation Biology, 20, 1445-1456. http://dx.doi.org/10.1111/j.1523-1739.2006.00491.x

Lussier, S. M., Enser, R. W., Dasilva, S. N., \& Charpentier, M. (2006). Effects of habitat disturbance from residential development on breeding bird communities in riparian corridors. Environmental Management, 38, 504-521. http://dx.doi.org/10.1007/s00267-005-0088-3

Marzluff, J. M. (2001). Worldwide urbanization and its effects on birds. In J. M. Marzluff, R. Bowman, \& R. Donnelly (Eds.), Avian ecology and conservation in an urbanizing world (pp. 19-47). Norwell, MA: Kluwer Academic Publishers. http://dx.doi.org/10.1007/978-1-4615-1531-9_2

Mason, J., Moorman, C., Hess, G., \& Sinclair, K. (2007). Designing suburban greenways to provide habitat for forest-breeding birds. Landscape and Urban Planning, 80, 153-164. http://dx.doi.org/10.1016/j.landurbplan.2006.07.002

McKinney, M. (2002). Urbanization, biodiversity, and conservation. Bioscience, 52, 883-890. http://dx.doi.org/10.1641/0006-3568(2002)052[0883:UBAC]2.0.CO;2

McKinney, M. (2006). Urbanization as a major cause of biotic homogenization. Biological Conservation, 127, 247-260. http://dx.doi.org/10.1016/j.biocon.2005.09.005

Nichols, J. D., Hines, J. E., Sauer, R., Fallon, F. W., Fallon, J. E., \& Heglund, P. J. (2000). A double-observer approach for estimating detection probability and abundance from point counts. The Auk, 117, 393-408. http://dx.doi.org/10.2307/4089721

Novak, A. B., \& Wang, Y. Q. (2004). Effects of suburban sprawl on Rhode Island's forests: A Landsat view $\begin{array}{llllll}\text { from } 1972 \text { to } 1000 . & \text { Northeastern }\end{array}$ http://dx.doi.org/10.1656/1092-6194(2004)011[0067:EOSSOR]2.0.CO;2

Odell, E. A., Theobald, D. M., \& Knight, R. L. (2003). Incorporating ecology into land use planning: the songbirds' case for clustered development. Journal of the American Planning Association, 69, 72-82. http://dx.doi.org/10.1080/01944360308976294

Oliver, A. J., Hong-Wa, C., Devonshire, J., Olea, K. R., Rivas, G. F., \& Gahl, M. K. (2011). Avifauna richness enhanced in large, isolated urban parks. Landscape and Urban Planning, 102, 215-225. http://dx.doi.org/10.1016/j.landurbplan.2011.04.007

Pidgeon, A. M., Radeloff, V. C., Flather, C. H., Lepczyk, C. A., Clayton, M. K., Hawbaker, T. J., \& Hammer, R. B. (2007). Associations of forest bird species richness with housing and landscape patterns across the USA. Ecological Applications, 17, 1989-2010. http://dx.doi.org/10.1890/06-1489.1

Porneluzi, P., Bednarz, J. C., Goodrich, L. J., Zawada, N., \& Hoover, J. (1993). Reproductive performance of territorial ovenbirds occupying forest fragments and a contiguous forest in Pennsylvania. Conservation Biology, 7, 618-622. http://dx.doi.org/10.1046/j.1523-1739.1993.07030618.x

Prevedello, J. A., \& Vieira, M. V. (2010). Does the type of matrix matter? A quantitative review of the evidence. Biodiversity and Conservation, 19, 1205-1223. http://dx.doi.org/10.1007/s10531-009-9750-z

Pyle, P., \& DeSante, D. F. (2011). Updates to four-letter and six-letter alpha codes based on revisions by the American Ornithologists' Union in 2011. Retrieved from http://www.birdpop.org/alphacodes.htm 
Radeloff, V. V., Hammer, R. B., \& Stewart, S. I. (2005). Rural and suburban sprawl in the US Midwest from 1940 to 2000 and its relation to forest fragmentation. Conservation Biology, 19, 793-805. http://dx.doi.org/10.1111/j.1523-1739.2005.00387.x

Rhode Island Geographic Information System. (2007). Rhode Island Geographic Information System Data Repository. Retrieved April 19, 2011, from http://www.edc.uri.edu/rigis/

Rhode Island Geographic Information System. (2011). Rhode Island Geographic Information System Data Repository. Retrieved March 23, 2012, from http://www.edc.uri.edu/rigis/

Rhode Island Statewide Planning Program. (1999). An analysis of Rhode Island land use. Rhode Island Statewide Planning Program Technical Paper 147.

Rhode Island Statewide Planning Program. (2001). Inventory of local zoning ordinances and land development regulations. Rhode Island Statewide Planning Program Technical Paper 148.

Rhode Island Statewide Planning Program. (2006). Land use 2025: Rhode Island state land use policies and plan. Rhode Island Statewide Planning Program, State Guide Plan Element 121, Report Number 109.

Riley, S. P. D., Busteed, G. T., Kats, L. B., Vandergon, T. L., Lee, L. F. S., Dagit, R. G., ... Sauvajot, R. M. (2005). Abundance of amphibians and invasive species in southern California streams. Conservation Biology, 19, 1894-1907. http://dx.doi.org/10.1111/j.1523-1739.2005.00295.x

Ritters, K. H., Wickham, J. D., O’Neill, R. V., Jones, K. B., Smith, E. R., Coulston, J. W., .. Smith, J. H. (2002). Fragmentation of continental United States forests. Ecosystems, 5, 815-822. http://dx.doi.org/10.1007/s10021-002-0209-2

Robbins, C. S., Dawson, D. K., \& Dowell, B. A. (1989). Habitat area requirements of breeding forest birds of the middle Atlantic states. Wildlife Monographs, 103, 3-34.

Robbins, C. S., Sauer, J. R., Greenberg, R. S., \& Droege, S. (1989). Population declines in North American birds that migrate to the neotropics. Proceedings of the National Academy of Sciences, 86, 7658-7662. http://dx.doi.org/10.1073/pnas.86.19.7658

Stein, S. M., McRoberts, R. E., Alig, R. J., Nelson, M. D., Theobald, D. M., Eley, M., ... Carr, M. (2005). Forests on the edge: housing development on America's private forests. General Technical Report PNW-GTR-636, Portland OR, US Department of Agriculture, Forest Service, Pacific Northwest Research Station. Retrieved from http://www.fs.fed.us/openspace/fote/fote-6-9-05.pdf

Stone Jr., B. (2008). Urban sprawl and air quality in large US cities. Journal of Environmental Management, 86, 688-698. http://dx.doi.org/10.1016/j.jenvman.2006.12.034

Syphard, A. D., Radeloff, V. C., Keeley, J. E., Hawbaker, T. J., Clayton, M. K., Stewart, S. I., \& Hammer, R. B. (2007). Human influence on California fire regimes. Ecological Applications, 17, 1388-1402. http://dx.doi.org/10.1890/06-1128.1

Theobald, D. (2005). Landscape patterns of exurban growth in the USA from 1980 to 2020. Ecology and Society, 10, 32. [online] URL: http://www.ecologyandsociety.org/vol10/iss1/art32/

Theobald, D. M., Miller, J. R., \& Hobbs, N. T. (1997). Estimating the cumulative effects of development on wildlife habitat. Landscape and Urban Planning, 39, 25-36. http://dx.doi.org/10.1016/S0169-2046(97)00041-8

Tu, J., Xia, Z., Clarke, K. C., \& Grei, A. (2007). Impact of urban sprawl on water quality in eastern $\begin{array}{llll}\text { Massachusetts, USA. Environmental } & \text { Management, } & \text { 40, }\end{array}$ http://dx.doi.org/10.1007/s00267-006-0097-x

US Department of Agriculture. (2009). Summary Report: 2007 National Resources Inventory. Natural Resources Conservation Service, Washington DC and Center for Survey Statistics and Methodology, Iowa State University, Ames, IA.

Van Horne, B. (1983). Density as a misleading indicator of habitat quality. Journal of Wildlife Management, 47, 893-901. http://dx.doi.org/10.2307/3808148

Van Rensburg, B. J., Peacock, D. S., \& Robertson, M. P. (2009). Biotic homogenization and alien bird species along an urban gradient in South Africa. Landscape and Urban Planning, 92, 233-241. http://dx.doi.org/10.1016/j.landurbplan.2009.05.002 
White, E. M., Morzillo, A. T., \& Alig, R. J. (2009). Past and projected rural land conversion in the US at state, regional, and national levels. Landscape and Urban Planning, 89, 37-48. http://dx.doi.org/10.1016/j.landurbplan.2008.09.004

Zhou, Y., \& Wang, Y. Q. (2007). An assessment of impervious surface areas in Rhode Island. Northeastern Naturalist, 14, 643-650. http://dx.doi.org/10.1656/1092-6194(2007)14[643:AAOISA]2.0.CO;2

\section{Copyrights}

Copyright for this article is retained by the author(s), with first publication rights granted to the journal.

This is an open-access article distributed under the terms and conditions of the Creative Commons Attribution license (http://creativecommons.org/licenses/by/3.0/). 